\title{
Scalability of MANET Routing Protocols for Heterogeneous and Homogenous Networks
}

\author{
Huda Al Amri, Mehran Abolhasan, Tadeusz Wysocki \\ Telecommunication and Information Research Institute, University of Wollongong, Wollongong, NSW 2522, Australia
}

\begin{abstract}
In Mobile Ad hoc Network (MANET), mobility, traffic and node density are main network conditions that significantly affect the performance of routing protocols. Much of the previous researches in MANET routing have focused on developing strategies, which suit one specific networking scenario. Therefore, there is no existing protocol that can work well in all different networking scenarios. This paper reviews characteristics of each different classes of routing protocols. Moreover, most of current routing protocols assume homogeneous networking conditions where all nodes have the same capabilities and resources. Although homogenous networks are easy to model and analysis, they exhibits poor scalability compared with heterogeneous networks that consist of different nodes with different resources. This paper presents extensive studies simulations for DSR, AODV, LAR1, FSR and WRP in homogenous and heterogeneous networks. The results showed that these which all protocols perform reasonably well in homogenous networking conditions, their performance suffer significantly over heterogonous networks.
\end{abstract}

\section{INTRODUCTION}

Mobile Ad hoc NETworks (MANET) are a group of wireless mobile nodes that have no fixed infrastructure. Therefore each node can act as router or an end-user node. Many routing protocols have been proposed to mange the communication on this kind of networking. Moreover, there are many issues that must be considered in constructing any routing protocol such as power consumption, reliable data delivery, and overheads and delays.

Recent work on MANET routing protocols have focused on achieving stability and reliability to reduce packet loss, communication overheads, and to increase data delivery ratio. Different approaches have been proposed to achieve those goals. Some of those focused on improving physical layer to provide reliable transmission, like diversity techniques, coding and Single Path Parallel Relays (SPPR) strategies [1-3]. Cooperation between link layer and network layer was another approach [3], where the state and the availability of the link on link layer were analyzed before calculating the routes [3]. Others expanded the existing protocols like AODV, LAR, and DSR by implementing the multipath strategy [4, 5]. However, mobility of the nodes has not been the main focus of those papers. We anticipate that several problems in MANETs arise due to the mobility such as high data delay and low packet delivery ratio. Hence, node mobility has to be considered in order to achieve high stability and reliability. Different strategies have been implemented in [6-8] to satisfy different degrees of mobility. On the other hand, most existing routing protocols have not been able to satisfy both scalability and mobility. Many routing strategies have been proposed to improve the performance of existing protocols or design new ones to deal with mobility or node density. In [8], Adaptive Cell Relay routing protocol (ACR) has been designed to deal with different density degree of the nodes to achieve high scalability. It uses two different routing strategies: the cell relay (CR) routing for dense networks, the large cell (LC) routing for sparse networks. It also monitors the node density changes to determine which routing strategy to apply according to the network density. A routing framework has been proposed in [6] to work on different mobility classes that are low, normal and fast. Mobility class was calculated using the proposed mobility metric referred to as "Stability". Stability is based on associativity that is defined as a time where the node can communicate with other nodes, and according to the stability value, a protocol is selected to route the packet. If a node is classified to be slow then a proactive protocol, like DSDV, will be used, if mobility class is normal then a reactive protocol like AODV will be applies, and the introduced RUNNER protocol will route the data if the mobility class is fast. In [9], two new protocols have been proposed to work with high mobility nodes in MANET. The idea behind these two protocols is that there is a group of mobile nodes which move throughout the entire network to receive and deliver data and control messages. These nodes are called the support nodes. One of the protocols is called Snake where the support nodes are predefined and then a leader election is carried out. The leader manages the movement of its group of support nodes in a form of snake movement. While each support nodes in RUNNER (second protocol) moves independently like a runner. However, the idea of support protocols cannot be applied in homogenous systems. Support nodes should have more capabilities and resources.

Few comparisons between different existing protocols have been published such as in [10, 11]. For example, in [10], AODV protocol and RUNNNER protocol have been evaluated. It has been found that AODV has higher data delivery ratio and lower data delay in dense network and low mobility of nodes. This is because AODV can reach destinations easily in such network conditions. On the other hand, RUNNER performs better in high mobility network where the support nodes are faster in delivering data. In [11], DSDV, AODV, and DSR have been compared in different scenarios of nodes mobility and traffic loads. The simulation results showed that reactive protocols (DSR and AODV) performed better than proactive protocols when nodes were moving. In addition, DSR works well with low traffic while AODV behaves better in higher traffic. A probabilistic model has been proposed in [12] to evaluate overhead of routing protocols of MANET. This model depends on network topology and data traffic parameters to estimate the number of control packets. In addition it can help identifying a protocol for particular situation. This model was tested by comparing it with existing simulations of AODV, 
DSR, and OLSR. Reactive protocols again performed better than proactive protocols when the mobility increases.

Most of current routing protocols assume homogeneous network conditions where all nodes have the same capabilities and resources. Although homogenous network are easy to model and analysis, they exhibits poor scalability compared with heterogeneous networks that consist of different nodes with different resources. Heterogeneous MANET comprise of mobile devices as Fig.1 that have different communications capability such as radio range, battery life, data transmission rate, etc. Moreover, in real world, some of MANET networks are obviously heterogeneous like military battlefield networks and rescue operations system. For instance, in a rescue operations system as Fig.2, there are limited mobile devices that are provided to individual rescuers, ambulances and police vehicles, and helicobacter. Limited mobile devices have lowest communication capabilities, while helicobacter is the most powerful communication device which forms backbone of the rescue team. Therefore, heterogeneity of nodes is another issue that needs to be considered in constructing and developing routing protocols for MANETs.

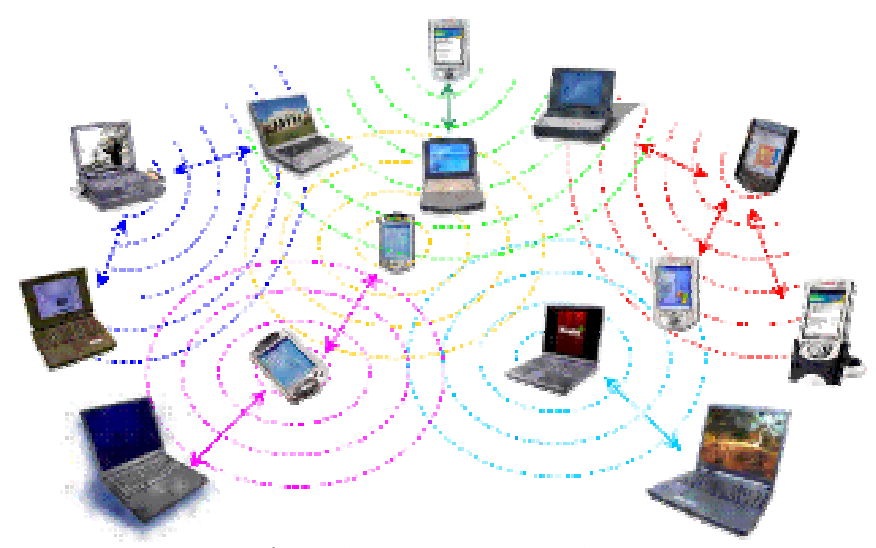

Figure. 1: Heterogeneous MANET.

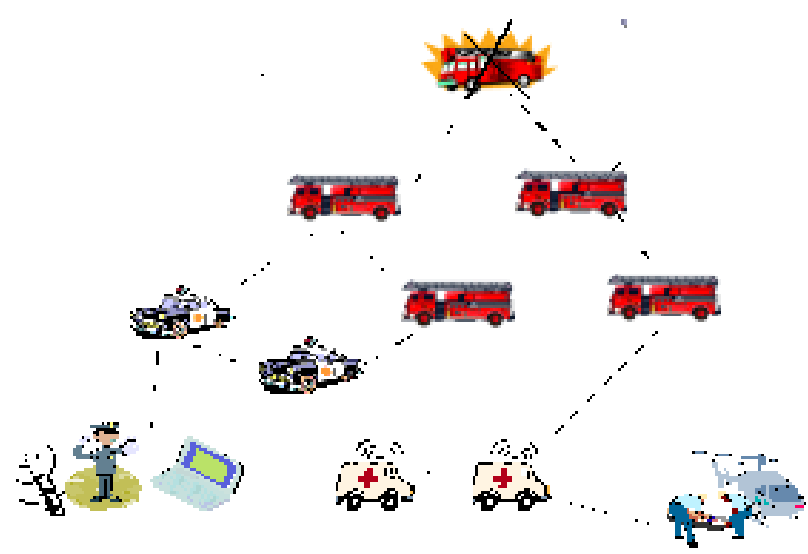

Figure. 2: Heterogeneous MANET in rescue operations System.

Recently, a few publications have introduced some strategies to develop routing protocols to accommodate heterogeneous MANETs. On-demand Utility-Based Routing Protocol
(OUBRP) strategy has been proposed in [13] to develop reactive routing protocols to efficiently utilize the heterogeneity of nodes. A utility-based route discovery algorithm is used to choose the richest nodes with highest level of resources during route discovery stage. The utility level of resources is reduced, if the route was not found. OUBRP reduces the number of re-broadcasting nodes. This strategy has been implemented over AODV. It has been found that this strategy improves routing discovery and reduces effect of route failure. In [14], scalability issue of OLSR in heterogeneous MANET has been studied. The study show that OLSR it does not differentiate distinct nodes with different communication capability and resources. This paper proposed a strategy to optimize OLSR to be scalable over large heterogeneous MANET. OLSR was improved by organizing nodes in hierarchal structure. Hierarchal OLSR (HOLSR) has eliminated overheads and reduces the size of routing table. With HOLSR, the nodes are organized in logical level, where nodes with lowest resources are in lower level. Each level has many clusters, where the cluster head is a powerful node with highest communication capability. HOLSR and flat OLSR have been compared in terms of control overhead, computations overhead, and end-to-end delay. HOLSR shows significant improvement to the performance of OLSR. Also it performs well in large heterogeneous MANET.

A new routing protocol was proposed in [15] to make use of heterogeneity in MANET. The entire network area was divided into cells with same size. The most powerful node in a cell acts as a gateway, where most the routing load goes through. This powerful node is called B-node. All B-nodes form the backbone of the entire networking. B-nodes reduce number of hops because they have high communication capability to transmit data.

Heterogeneous MANETs have the potential of reducing the amount of power used at user nodes. In [16] author state that supply of power in heterogeneous wireless ad hoc networking can affect the lifetime of network. They proposed a cross- layer for Device Energy-Load Aware Relaying (DELAR) strategy to utilize powerful nodes. This strategy suggested having a schedule to use different transmission powers in different periods. They also proposed "mini-routing" and Asymmetric MAC (A-MAC) to support link level acknowledgements with unidirectional links. The simulation of DELAR showed that this strategy can reduce power consumption and increase lifetime of network.

The common approach to dealing with heterogeneity of nodes in previous papers [13-15] is to assign most of the routing load for the powerful nodes, as they possess more resources and communication capabilities. Consequently, this approach eliminates number of hopes and can reduce delay. However, this strategy may create critical problems if the powerful nodes go off-line. For example, in a battlefield network scenario, if a vehicle or a tank possessing more powerful communication capabilities was destroyed, then the communication with others including soldiers and vehicles 
could be lost. Most publications have not considered this situation. There should be alternative strategies to recover any fault on powerful nodes that have been assigned as routers.

Up to now, several reviews have been published which described the functionality and theoretical performance of MANET routing protocols. For example, in [17, 18] routing protocols for MANETs have been revised and classified according their scalability. However, no study has attempted to evaluate the performances of current routing protocols in heterogeneous MANETs. In this paper, different classes of MANET routing protocols are reviewed. A suitable class of routing protocols is suggested to perform well in a particular network conditions. Additionally, the performances of DSR, AODV, LAR1, FSR and WRP are compared by simulating them in homogenous and heterogeneous MANET.

In section two, a review of the key features of existing routing protocols is summarized. The performance of these protocols is analyzed in section three. Section four describes our simulations of different protocols in homogenous and heterogeneous MANET. Section five discusses our results. Last section concludes this paper.

\section{REVIEW OF MANET ROUTING PROTOCOLS}

Routing protocols for MANETs have been classified according to the strategies of discovering and maintaining routes into three classes: proactive, reactive, and hybrid [17]. Of course, each routing protocol reacts differently to node mobility and density. A routing protocol for MANETs is usually evaluated in terms of performance metrics that are endto-end delay, overhead, throughput and data delivery ratio. This section outlines the main features of each class. Also, a brief summary about the protocols that have been used in simulations is given.

Proactive Routing Protocols

Proactive routing protocols acquire routing information periodically and store then in one or more routing tables. The differences among the protocols in this class are routing structure, number of tables, frequency of updates, use of hello messages and the existence of a central node. Therefore, each protocol reacts differently to topology changes. Flooding of routing information is the mechanism that is often used to discover and update routes. However, it is common that proactive protocols generate more control traffic and overhead than other protocol classes because of periodic updating which increases as the number of nodes increases. Moreover, they extensively use memory for storing those tables. Examples proactive routing protocols are: Wireless Routing Protocol (WRP) and Fisheye State Routing (FSR). With WRP [19], each node maintains four routing tables. As the network increases, this protocol consumes significant amount of memory. Moreover, hello messages are used to ensure the connectivity with neighbors. Consequently, this will consume more bandwidth and power.
FSR [20] is another proactive protocol. This protocol updates network information frequently for nodes that are within its scope only. Therefore, it is more scalable than WRP. However, FSR is not adaptable to high mobility.

Reactive Routing Protocols

Reactive routing protocols discover or maintain a route as needed. This reduces overhead that is created by proactive protocols [17, 21]. Flooding strategy is used to discover a route. Reactive routing protocols can be classified into two groups: source routing and hop by hop routing. In source routing, data packet headers carry the path to destination. Hence, intermediate nodes do not care about maintaining the routing information. On the other hand, this kind of protocols may experience high level of overhead as the number of intermediate nodes increases. Also they have a higher chance of a route failure. Packets in the second group of reactive protocols have to carry only destination and next hop addresses which means that nodes have to maintain and store routing information for active routes. In general, reactive protocols suffer from delay because of the route discovery process. Ad hoc On-Demand Distance Vector (AODV) [17, 22] and Dynamic Source Routing protocol (DSR), and Location-Aided Routing (LAR) are well-known reactive routing protocols.

AODV is a hop-by-hop routing protocol, which introduces a more dynamic strategy to discover and repair route when compared to DSR. Destination sequence numbers are used to avoid the problem of infinite loops. AODV maintains only active routes to reduce overheads and control traffic. This protocol is applicable for different levels of node density, mobility and loads. It is suitable for scenarios with moderate mobility and density networks.

DSR is a reactive source routing protocol [17, 23]. It discovers routes on demands using route discovery and maintenance strategy. Multiple routes are applied to achieve load balancing and to increase robustness. DSR can operate well with high mobility nodes because it can recover from routes failure quickly. It can support up to one hundred node which means it can work well over medium network density.

LAR [24] uses GPS information to detect the location of nodes. This reduces overhead due to flooding. This protocol has two strategies for route discovery. Firstly, it limits the RREQ propagation for define area (i.e. Request Zone). Secondly, stores the coordinates of destination node where the route request packets travel toward the destination coordinators. This reduces overheads. However, each node must have GPS.

Hybrid Routing Protocols

Hybrid protocols exhibit both reactive and proactive features. Proactive strategy is used to discover and maintain routes to near by nodes, while routes to far away nodes are discovered reactively. Consequently, overheads and delay that are introduced by proactive protocols and reactive protocols, respectively, are minimized. Hybrid protocols have been known to be more scalable than others fewer nodes take part in routing and topology discovery. In Zone Routing Protocol 
(ZRP), the nodes are grouped into zones. Communications between nodes depend on their locations in the zone. Another example of hybrid protocol that can adapt to changes in node density and mobility is Scalable Location Update Routing Protocol (SLURP). It uses GPS information to mange node location and eliminates global routing. Each node is associated with a home region and sends its new location to its home region as it moves .Hence, when a route is required, the source node only have to query the home region of the destination. This protocol is suitable for large networks where the number of nodes and their mobility are high $[17,25]$.

Hierarchal and Geographic Routing Protocols

MANET routing protocols can be divided also according to routing structures into flat routing protocols, hierarchal routing protocols, and geographic position information assisted routing protocols $[18,26]$. Each protocol routes data proactively or reactively or uses the combination of the two strategies. Flat protocols can be tables driven (proactive) like DSDV and on demand protocols (reactive) like DSR. Those protocols have been described previously.

The idea of wireless hierarchal routing protocols is to group mobile nodes to reduce the area of flooding. The nodes are grouped in terms of clusters, trees or zones where there is a leader that manages routing in its area. Each node has different functionality depending on its location inside the group or outside it. This strategy reduces the size of routing tables and the routing information [18]. Example of wireless hierarchal routing protocols is the ZRP. The advantages of those protocols are in the reduction of overheads and improved scaling of large networks compared to flat routing protocols. However, when node mobility is high, hierarchal routing may introduce more overhead due to cluster re-calculation. In addition, a cluster head is a critical node and communication breaks if it goes down.

Geographic position information assisted routing protocols improve routing by using Global Position System (GPS) receivers built into the nodes to get their location information [18]. Those protocols route the data using Geographic Addressing and Routing (GeoCast) where messages are sent to all nodes in specific geographical area. GeoCast uses the geographical information rather than logical addresses. Geographical information about nodes eliminates propagation of routing information. Hence, geographical protocols have more efficiency in adapting to changes in node density compared to other protocols. Examples of geographic routing are DREAM and SLURP. However, mapping address to location produces more overheads. In addition, using GPS consumes the power of a mobile node.

\section{COMPARISON OF DIFFERENT CLASSES OF ROUTING PROTOCOLS FOR MANETS}

In this section, comparison of proactive, reactive and hybrid protocols is outlined by combining their published theoretical performance [27] [12]. That comparison is further verified through the published simulation results $[6,11,27-30]$. Based on that comparison, a suitable class of routing protocols is suggested to perform well in a particular network conditions.

\section{A. Theoretical and model based analysis}

Proactive protocols are the oldest protocols that have been derived from wired network routing protocols to work in the wireless environment. Therefore, they possess many features of wired routing protocols like routing tables that are used to keep the routing information, which are periodically updated even if not needed. As the node moves, there is a flooding of packets containing the topology changes causing high overheads. Hence, in general, proactive protocols produce more overheads resulting in a lower throughput in case of high mobility as illustrated in theoretical and model based analysis below.

In order to compare the protocols, the following set of parameters is usually defined:

$\mathrm{N}=$ number of nodes.

$\mathrm{L}=$ average path length (in hops).

$\mathrm{R}=$ average number of active routes per node.

$\mu=$ average number of link breakage per second (reflect mobility degree).

$\alpha=$ route activity, which gives how the frequently the node is changing its destination.

$\rho=$ route concentration factor that monitors the traffic hotspots in MANET.

Proactive, reactive and hybrid protocols have been evaluated theoretically in [27]. It has been found that asymptotic overhead for proactive is $\mathrm{O}\left(\mathrm{N}^{1.5}\right)$ due to the process of maintaining and forwarding tables to keep periodic updates. In reactive protocols, route requests and reply messages create overhead of cost $\mathrm{O}\left(\mathrm{N}^{2}\right)$, while in hybrid protocols this is $\mathrm{O}\left(\mathrm{N}^{1.66}\right)$. The number of packets that are produced by proactive protocols per second is $\mu^{*} \mathrm{~L}^{*} \mathrm{~N}^{2}$ while for reactive protocols is $\left(\alpha+\rho^{*} R^{*} \mu\right)^{*} L^{*} N^{2}$. Reactive is found to be better than proactive if $\mu^{*} \mathrm{~L}^{*} \mathrm{~N}^{2}>\left(\alpha+\rho^{*} \mathrm{R} * \mu\right) * \mathrm{~L}^{*} \mathrm{~N}^{2}$. It has been concluded in [25] that proactive protocols can be used mostly in static or quasistatic networks, reactive protocols are preferred in more dynamic networking, while hybrid protocols are more efficient in adapting to changes in network conditions.

Analytical model that compared control overhead with mobility and data traffic for proactive and reactive protocols for MANETs has been also presented in [12]. It has been found that number of packets produced by optimized reactive protocols in MANET is $o_{r} \mu \mathrm{aLN}^{2}$ and $o_{\mathrm{p}} \mu \mathrm{ANpN}^{2}$ for optimized proactive protocols, where

$\mathrm{o}_{\mathrm{r}}=$ route request optimization factor.

$\mathrm{AN}_{\mathrm{p}}=$ active next hops ratio.

$\mathrm{a}=$ number of active routes per node (activity).

$\mathrm{o}_{\mathrm{p}}=$ broadcast optimization factor.

As a result of comparing those two approaches with existing simulations, it has been observed that OLSR is more scalable than DSR. Moreover, rough high mobility asymptotic for both classes have been compared. It has been found that reactive protocols are better than proactive in high mobility if reactive protocols use routes that do not share links. 
Hierarchal routing protocols, geographic position information assisted routing protocols, and hybrid routing protocols are more adaptable to various node destination than flat protocols [17, 18]. In [17], hierarchal routing protocols have been found to be more scalable than flat protocols because they limit the propagation area by structuring the network nodes. However, overheads are increasing with those routing schemes due to location management. Therefore, hierarchal protocols are suitable in scenario like high density but low mobility. Geographic routing protocols also perform well in high density because of the simplicity of location management localized route discovery.

\section{B. Simulation Observations}

MANET routing protocols are commonly evaluated according to performance metrics such as: delay, delivery ratio, and overheads. Delay is the delay of data processing and queuing in intermediate nodes. Delay increases usually as mobility increases in all different classes of routing protocols. The delivery ratio is the ratio of the number of received packets at the destination to the number of packets that are sent by the source node. This ratio usually decreases as mobility increases. The last metric is the overhead consuming the network bandwidth, which is often high as nodes increase their speed. Adaptable protocol to particular scenario of density and mobility has lower delay and overhead and higher delivery ratio. Several simulations have been carried out to compare different protocols from different classes in different scenarios of nodes mobility and density [6, 11, 27-30].

The results of these simulations indicated that proactive protocols have higher overhead than reactive and hybrid protocols in terms of mobility and density while they have smaller delay than reactive ones. On the other hand, reactive protocols have lower delay than hybrid protocols. Although it is noticed that as the density increases and the mobility decreases, the delivery ratio increases. Proactive protocols have better delivery ratio but hybrid protocols have the best delivery ratio. Hence, they perform better in high density networks.

In [30], several simulations of four protocols have been carried out using GloMoSim simulator. These protocols were distance vector (DV), DSR and AODV as reactive protocols, and WRP as proactive protocol. The simulations have been run under different network conditions like different mobility degrees and different nodes density. It has been found that DSR has highest delay, while WRP has the lowest overhead as mobility increases.

To conclude what we have outlined theoretically and from existing simulations, proactive protocols class perform well in network with low mobility nodes. However, this class can adapt different node density, because they include hierarchal and geographical routing protocols. Moreover, hierarchal, geographic and, and hybrid routing protocols, have been more flexible with high density networks. Therefore, they can operate with medium and high density. In medium density and mobility, reactive protocols can work well.

\section{SIMUlation MODEL}

In this section we present simulations that have been carried out to compare the performances of different protocols from different classes in heterogeneous and homogenous MANET. In homogenous MANETs, all nodes have same capabilities and resources while with heterogeneous MANET different nodes have different resources like transmission range and power saving.

We preformed the simulations using the GloMoSim [31] package. Each simulation run for 900 s with different values of seeds. There was a 50-node network on a 1500x300 grid, a 100 -node network on a 2200x600 grid, and a 200-node network on a 3000x1000 grid. Random way point was used as mobility model with eight different values of pause times that were $0,50,100,200,300,500,700$ and 900. Speeds of the nodes were varied from 1 to $20 \mathrm{~m} / \mathrm{s}$. Different traffic loads flows have been created between random pairs of nodes. There were 10 flows from source to destination over 50-node, 110 flows over 100-node and 210 flows over 200-node. Constant Bite Rate (CBR) was used to generate data traffic of rate 4 packets per second. Each packet was 512 bytes and transmitted at $250 \mathrm{~ms}$ intervals.

IEEE 802.11 was used as MAC protocol with constant transmission bandwidth of $2 \mathrm{Mbps}$. The transmission power was $15 \mathrm{dbm}$ for all nodes in homogenous network. In heterogeneous MANET, nodes have different transmission powers (1-20 dbm) and receiver powers (-81.0 - -110.0 dbm).

The simulations run five different protocols that were DSR, AODV, LAR1, FSR and WRP. Packet delivery ratio (PDR), End-to-End Delay and packet loose percentage, control overhead and hop counts were used as performance metrics of each protocol.

\section{RESULTS}

In this section, we present the results of simulating DSR, AODV, FSR, LAR1 and WRP with different number of nodes within heterogeneous and homogenous networks. DSR, AODV and LAR1 were simulated with 50, 100 and 200 nodes while FSR has simulated with 50 and 100 nodes and WRP with 50 only. This is because FSR and WRP are not scalable to large number of nodes.

Fig 3 (a-e) shows End-to-End delay of each protocol. This graph shows that all protocols behave differently with heterogeneous nodes. The delay with 50 and 100 homogenous nodes are nearly constant. They all have higher delay in heterogeneous MANET comparing with homogenous MANET and with same number of nodes. For example, AODV has delay less than 0.2 with 50 homogenous nodes while it is more than 0.3 with 50 heterogeneous nodes. Also, the delay is extremely high with 200 heterogeneous nodes for all protocols. DSR behaves better in heterogonous MANT than other protocols do where delay is very low except for 200 heterogeneous nodes as shown in Fig3(b). FSR has high delays with 100 nodes for both homogenous and heterogeneous networks as in Fig.3(c). Fig.3 (d) illustrates the delay for 
LAR1, all delays of different number of nodes in heterogeneous MANET are the highest where it varies from 6 to 10 comparing with other protocols. All these reflect that current routing protocols are not adaptable for heterogeneous nodes.

Fig 4 (a-e) illustrate the packet delivery ratio (PDR) of different protocols with homogenous and heterogeneous networks. PDR of all protocols is nearly 1 in homogenous networking with different number of nodes. It decreases with heterogeneous networking. The difference between the PDR in homogenous and in heterogeneous networks with same number of nodes is higher with proactive protocols like FSR and WRP. This difference is $20 \%$ for reactive protocols while it is nearly $60 \%$ with proactive protocols. This shows that those protocols do not make use of the different resources that different nodes have it.

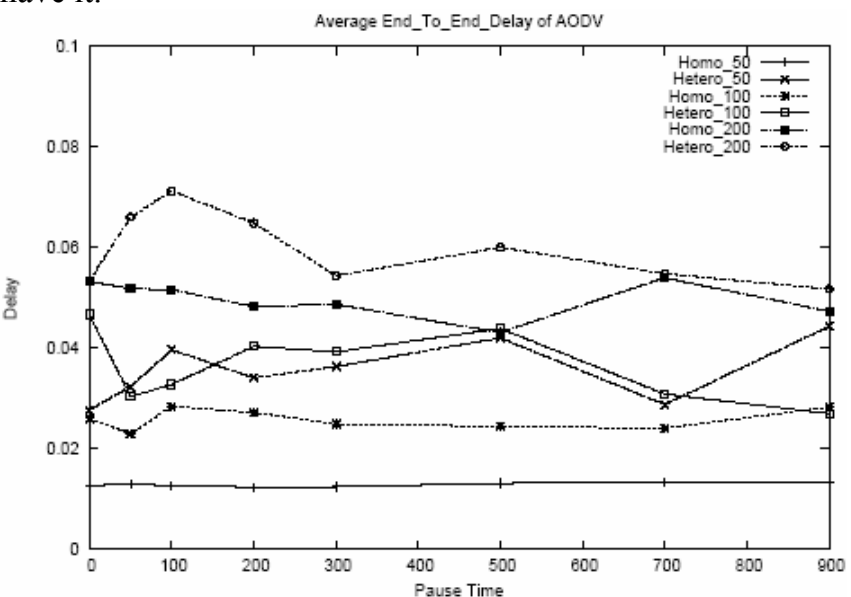

(a): End-to-End Delay of AODV for 50, 100, and 200 homogenous and heterogeneous nodes.

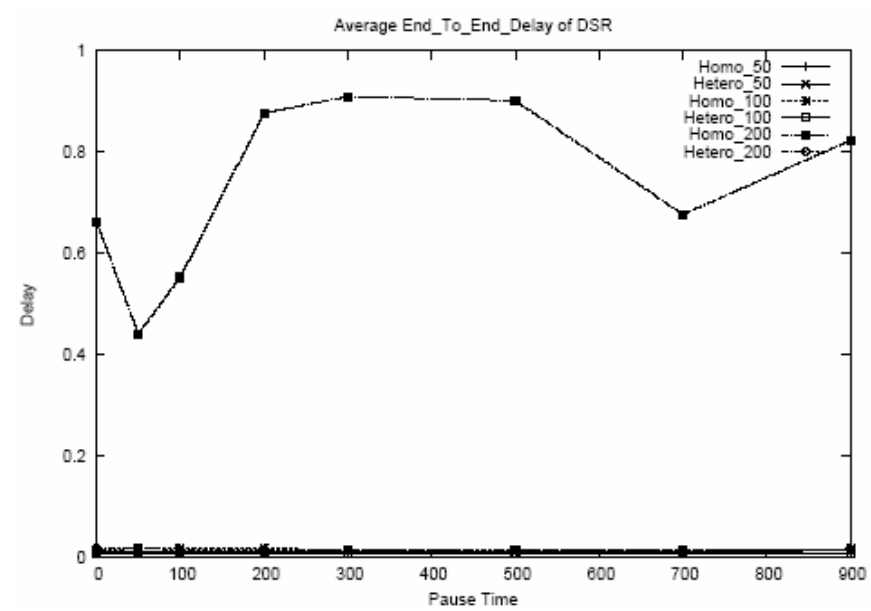

(b): End-to-End Delay of DSR for 50, 100, and 200 homogenous and heterogeneous nodes.

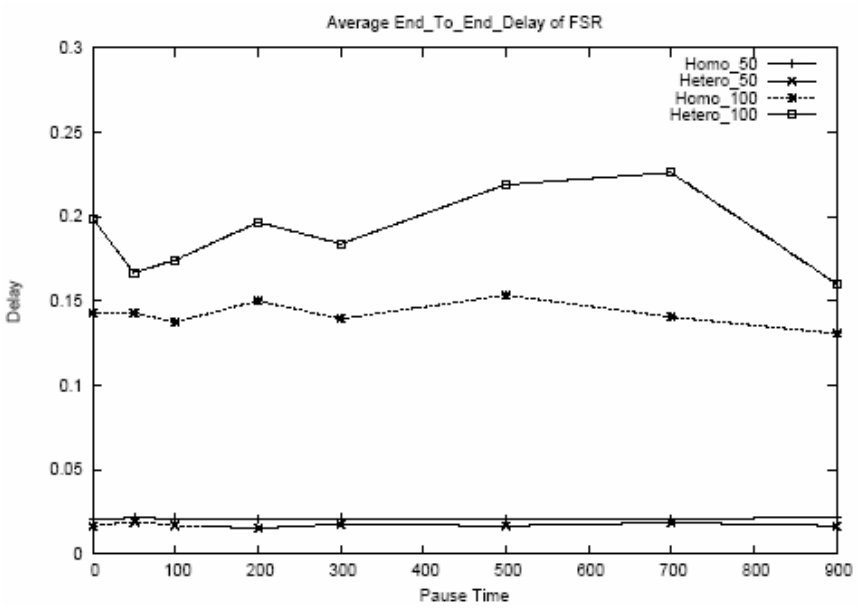

(c): End-to-End Delay of FSR for 50 and 100 homogenous and heterogeneous nodes.

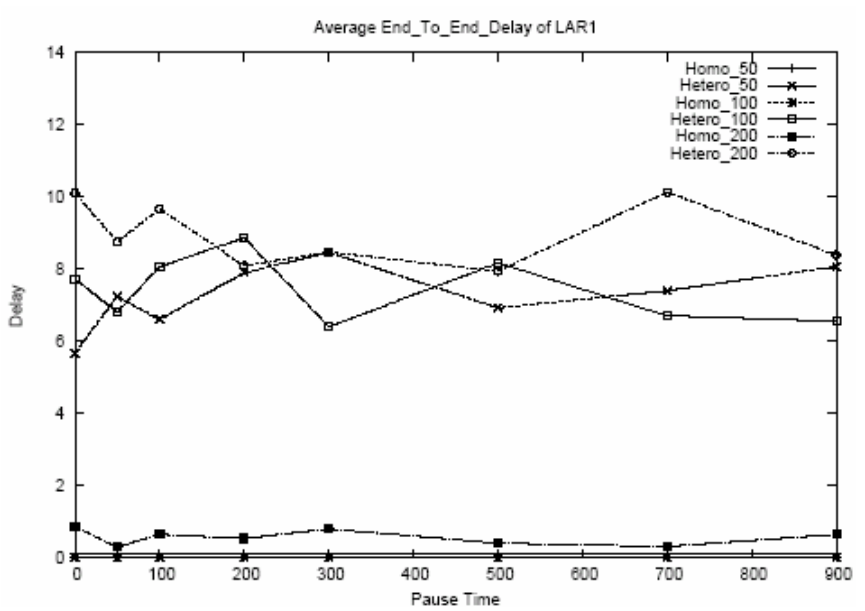

(d): End-to-End Delay of LAR1 for 50, 100, and 200 homogenous and heterogeneous nodes.

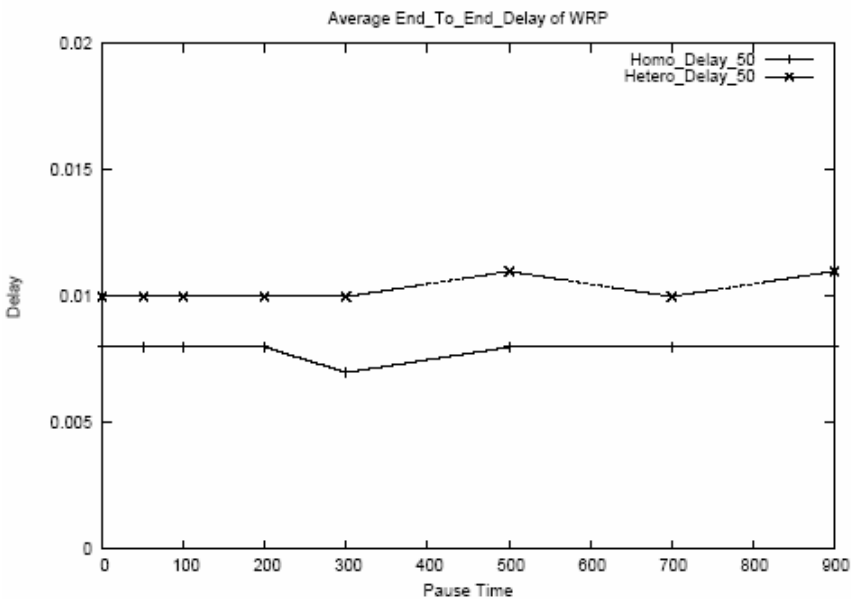

(e): End-to-End Delay of WRP for 50 homogenous and heterogeneous nodes.

Figure.3. (a-e): End-to-End Delay of AODV, DSR, FSR, LAR1, and WRP for both homogenous and heterogeneous networks. 
Packet loss percentage is illustrated in Fig 5(a-e). In homogenous network, the rate of packet loss is very low compared to heterogeneous networking. Packet loss percentage in heterogeneous networking with reactive protocols is between 20 and 25 while it ranges from 60 to 70 for proactive protocols.

Overheads are illustrated in Fig 6(a-e). Overhead is higher too with heterogeneous networking. 200 nodes with heterogeneous networking has very high overhead with AODV, DSR and LAR1. This illustrates the scalability issue. Proactive protocols as expected have the highest overhead in both homogenous and heterogeneous networking. This is because of periodical updating of routing information.

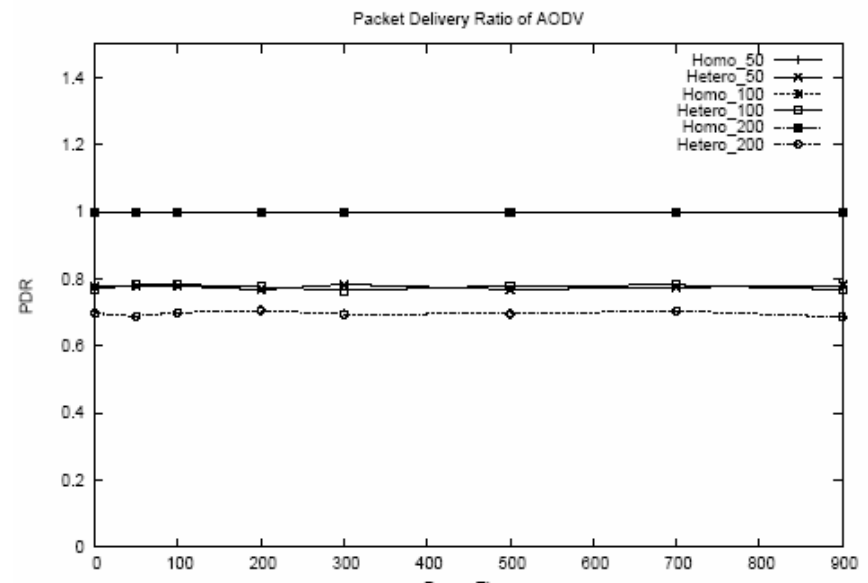

(a): Packet Delivery Ratio of AODV for 50, 100, and 200 homogenous and heterogeneous nodes.

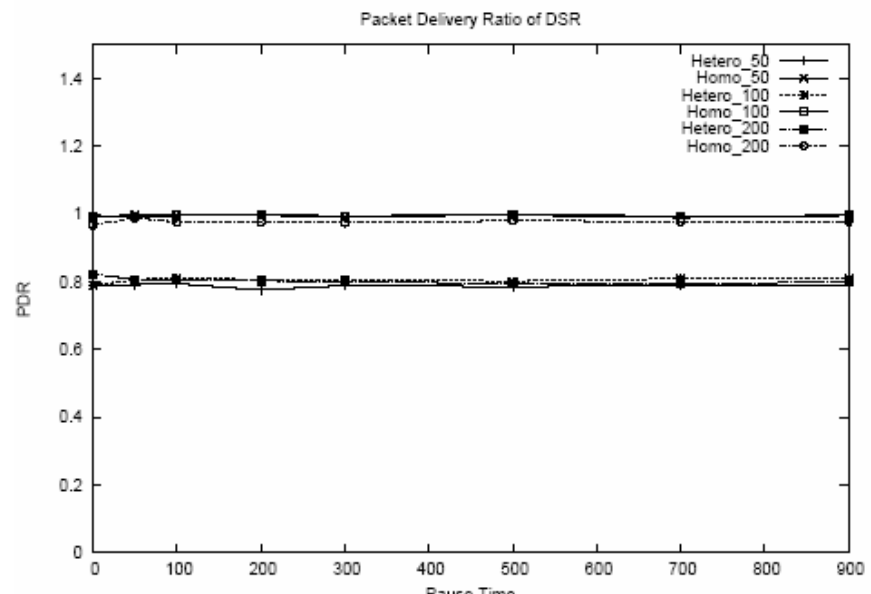

(b): Packet Delivery Ratio of DSR for 50, 100, and 200 homogenous and heterogeneous nodes.

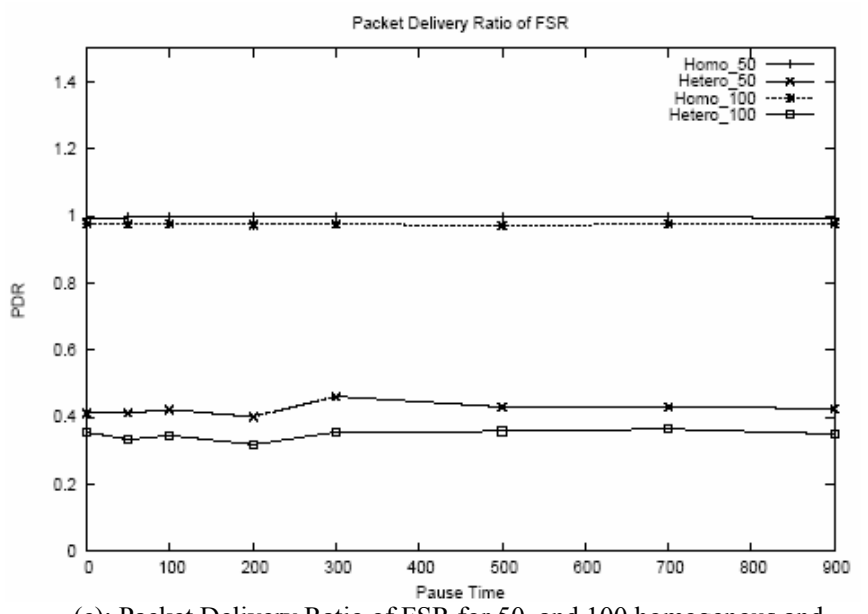

(c): Packet Delivery Ratio of FSR for 50, and 100 homogenous and heterogeneous nodes.

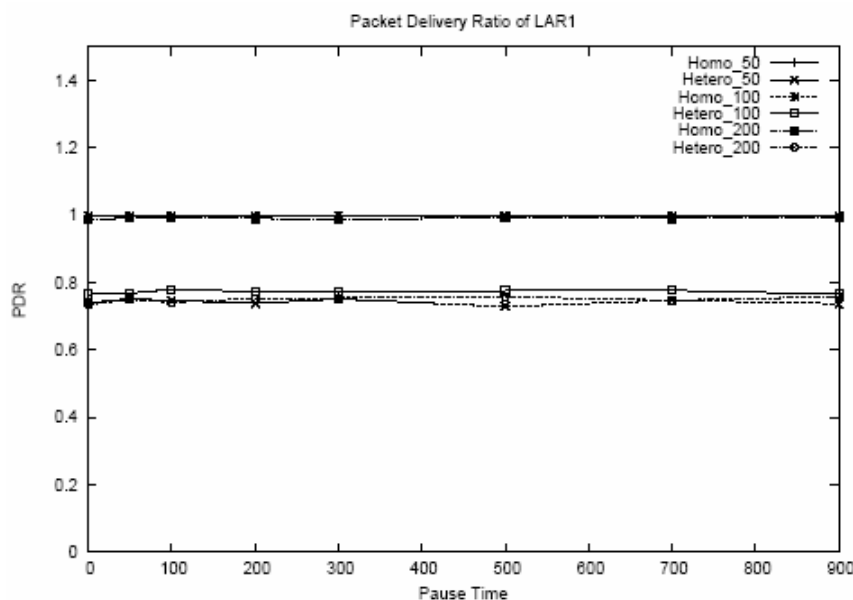

(d): Packet Delivery Ratio of LAR1 for 50, 100, and 200 homogenous and heterogeneous nodes.

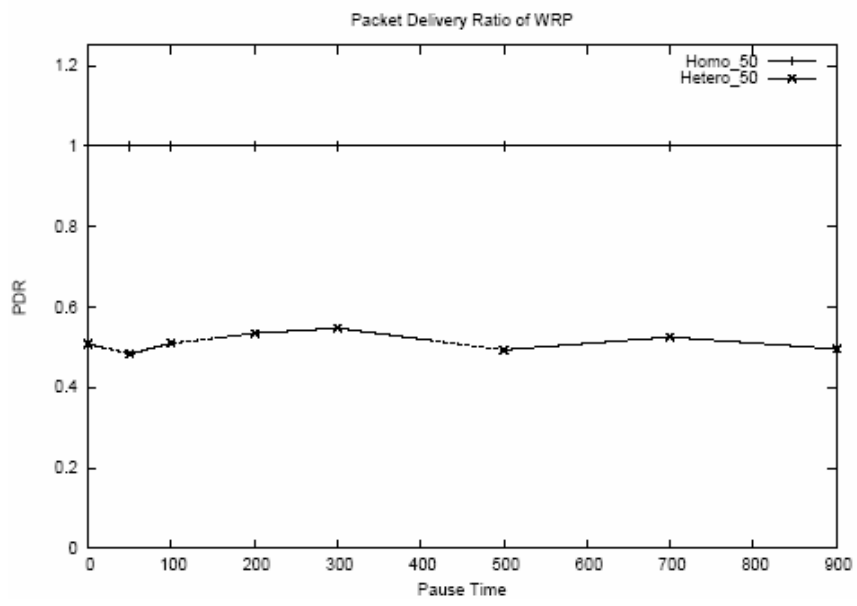

(e): Packet Delivery Ratio of WRP for 50 homogenous and heterogeneous nodes.

Figure.4. (a-e): Packet Delivery Ratio of AODV, DSR, FSR, LAR1, and WRP for both homogenous and heterogeneous networks. 
Generally, most protocols behave inefficiently and unexpectedly in heterogeneous networks. One of the problems that cause misbehaving is unidirectional link. Some protocols support only bidirectional link between two similar nodes.

Unidirectional link problem is shown in Fig 7, where node B has higher transmission range than node $\mathrm{A}$. Therefore

$\mathrm{B}$ includes $\mathrm{A}$ in its transmission range while $\mathrm{A}$ does not include $\mathrm{B}$. Consequently, the link between $\mathrm{B}$ and $\mathrm{A}$ is unidirectional from B to A only. However, AODV assumes all links between two nodes are bidirectional which gives incorrect routing information. Therefore, this incorrect information creates large delay and packet loss in heterogeneous networking.

However, in heterogeneous networking, there are nodes which have high transmission range to connect to large number of nodes. Therefore, the number of neighbor nodes increases. Hence, as network size increases, powerful nodes will consume more memory and bandwidth in storing neighbor tables and updating routing information. Therefore, proactive protocols might experience higher percentage of packet losing and lower PDR.

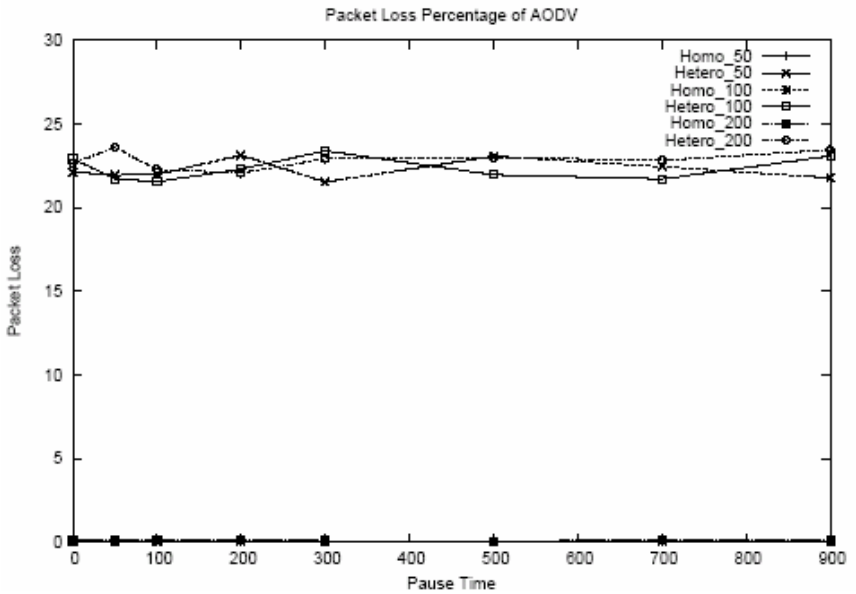

(a): Packet Loss Percentage of AODV for 50, 100, and 200 homogenous and heterogeneous nodes.

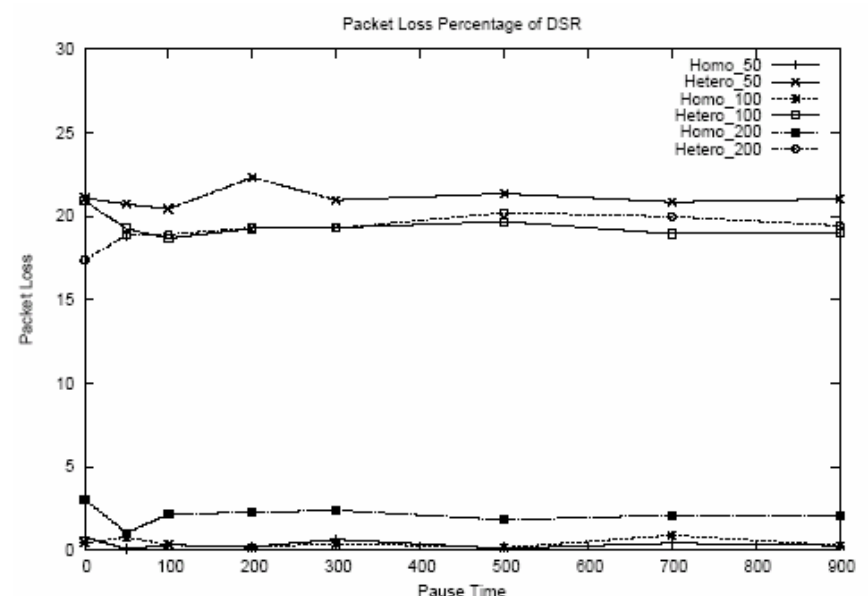

(b): Packet Loss Percentage of DSR for 50, 100, and 200 homogenous and heterogeneous nodes.

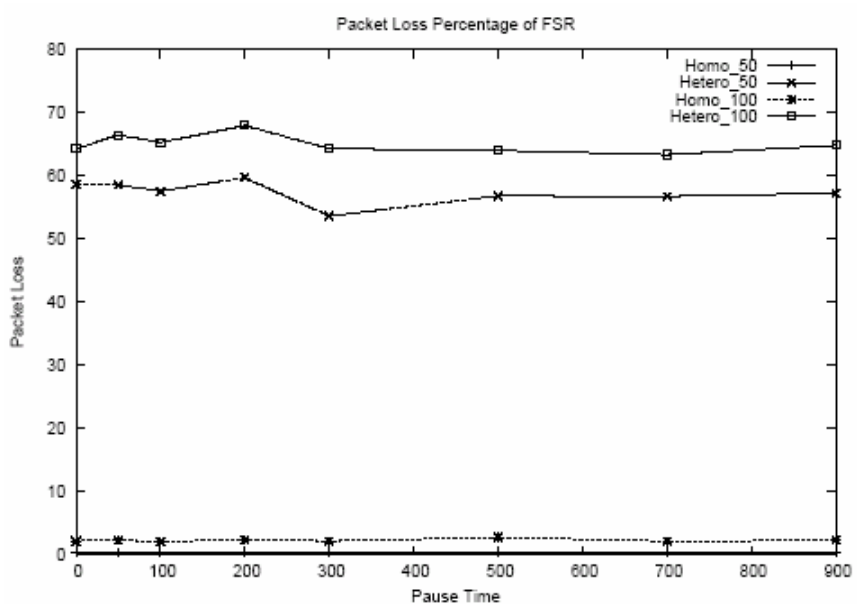

(c): Packet Loss Percentage of FSR for 50, and 100 homogenous and heterogeneous nodes.

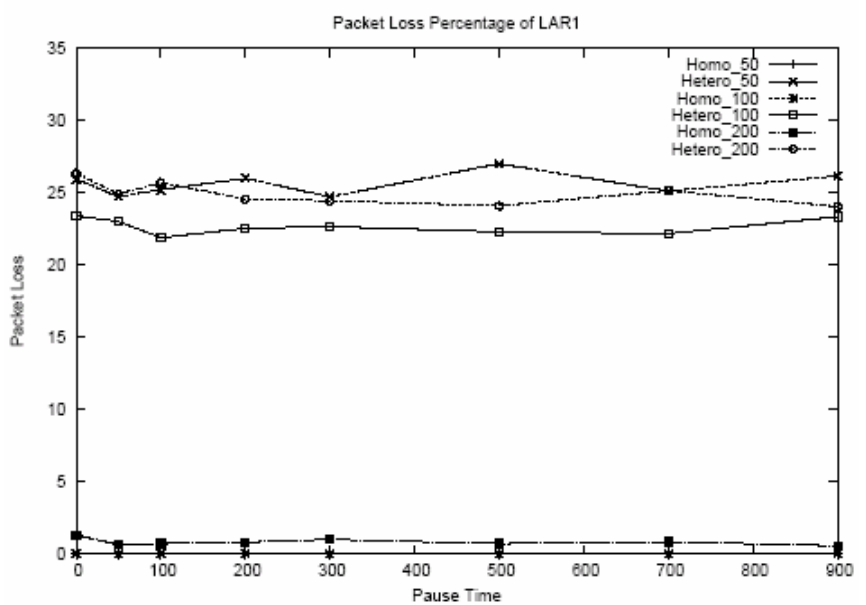

(d): Packet Loss Percentage of LAR1 for 50, 100, and 200 homogenous and heterogeneous nodes.

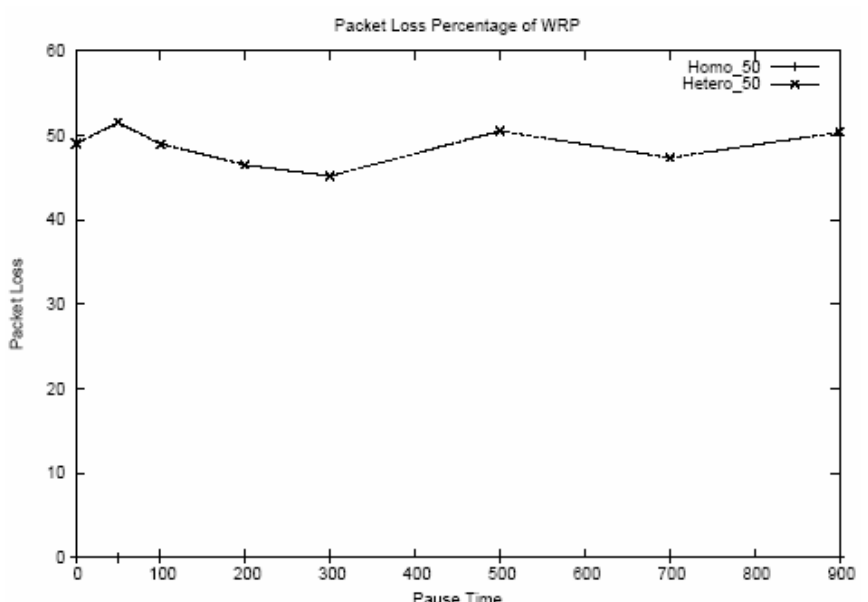

(e): Packet Loss Percentage of WRP for 50 homogenous and heterogeneous nodes.

Figure.5. (a-e): Packet Loss Percentage of AODV, DSR, FSR, LAR1, and WRP for both homogenous and heterogeneous networks. 


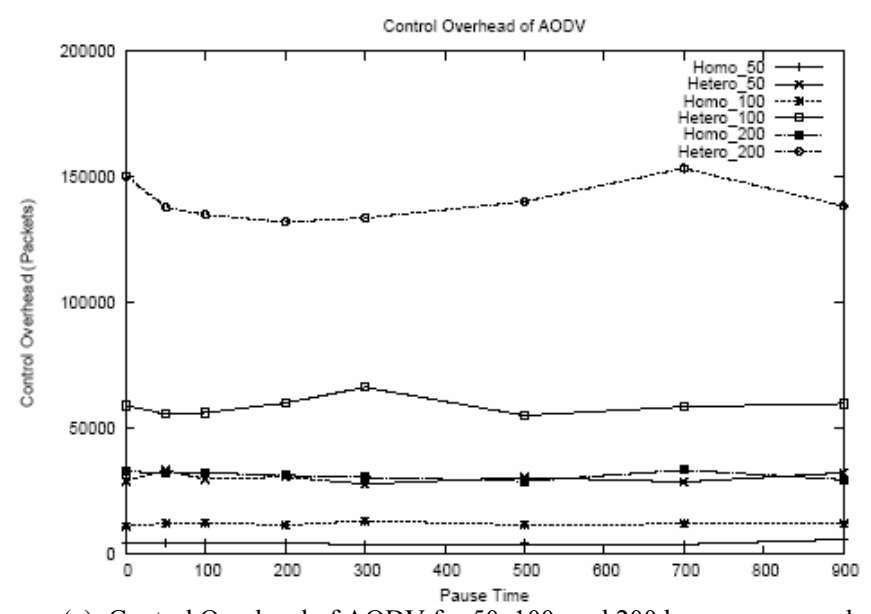

(a): Control Overhead of AODV for 50, 100, and 200 homogenous and heterogeneous nodes.

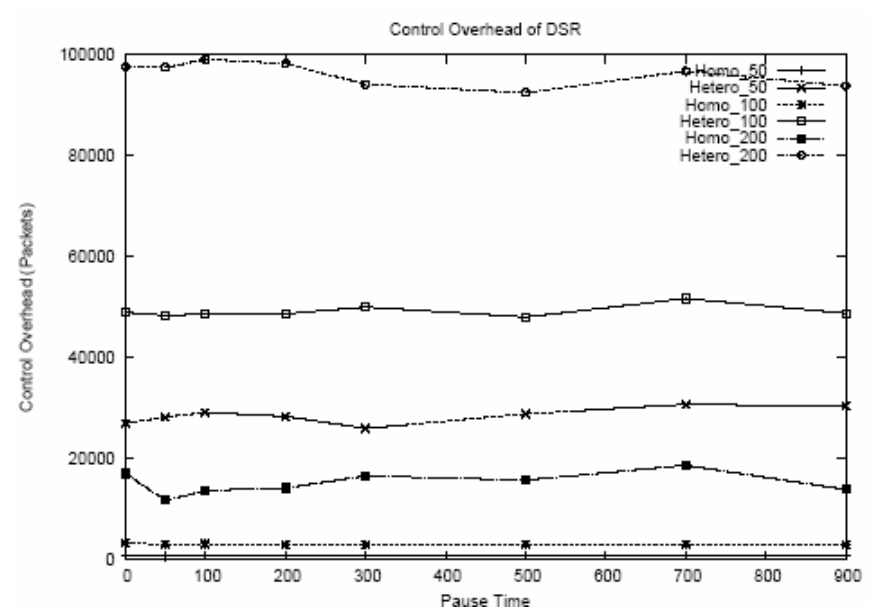

(b): Control Overhead of DSR for 50, 100, and 200 homogenous and heterogeneous nodes.

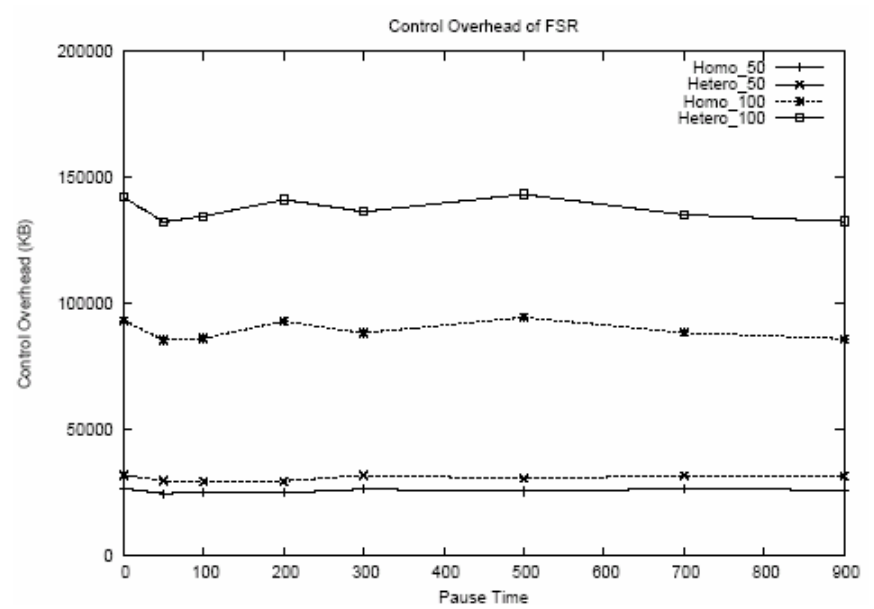

(c): Control Overhead of FSR for 50, and 100 homogenous and heterogeneous nodes.

Figure.6. (a-e): Control Overhead AODV, DSR, FSR, LAR1, and WRP for both homogenous and heterogeneous networks.

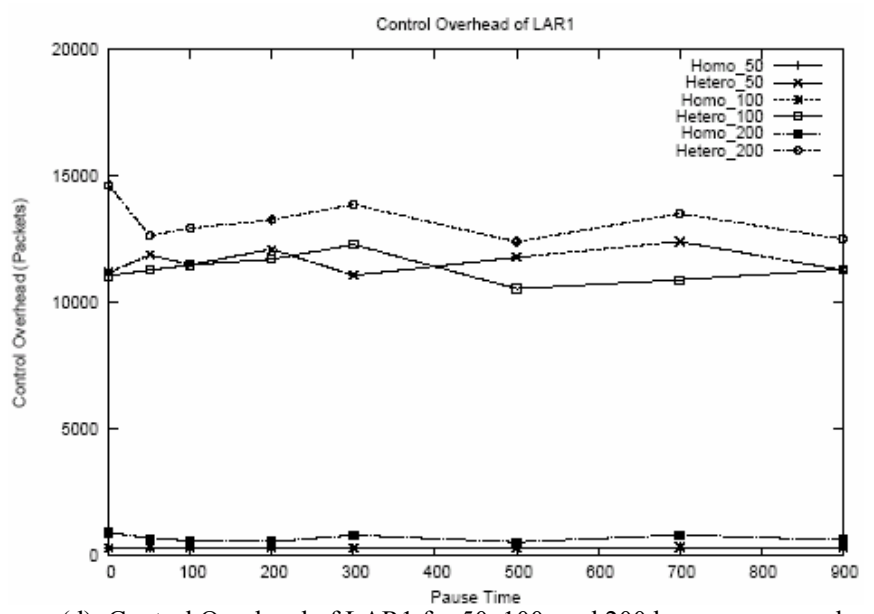

(d): Control Overhead of LAR1 for 50, 100, and 200 homogenous and heterogeneous nodes.

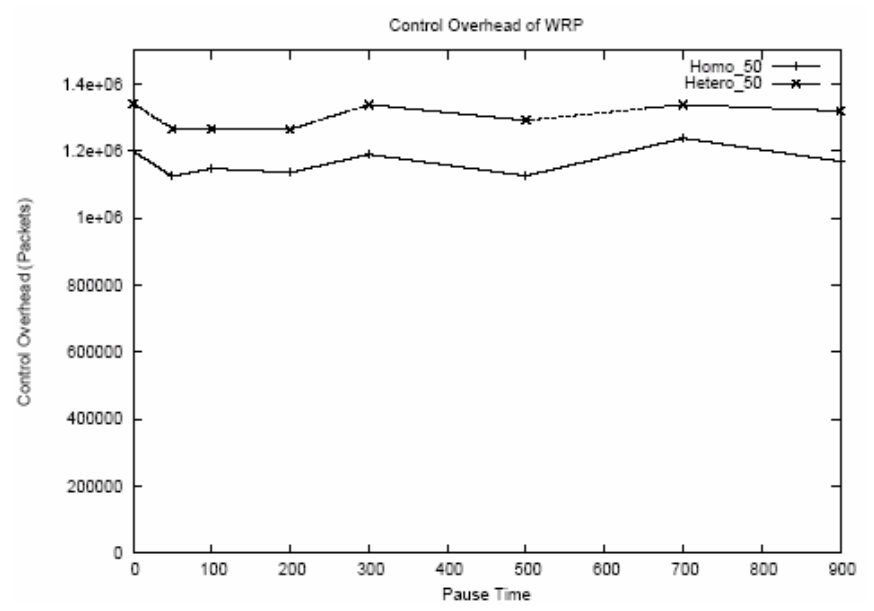

(e): Control Overhead of WRP for 50 homogenous and heterogeneous nodes.

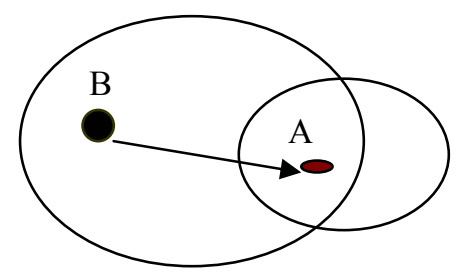

Figure.7: Unidirectional Link Problem in Heterogeneous MANET.

\section{CONCLUSION}

In Mobile Ad hoc Network (MANET), mobility, traffic and node density are main network conditions that significantly affect the performance of the network. This issue has been reviewed in this paper. In addition, most of current routing protocols assume homogeneous network conditions where all nodes have the same capabilities and resources. Although homogenous networks are easy to model and analysis, they exhibits poor scalability compared to heterogeneous networks, which consist of different nodes with different resources. In this paper, different simulations have been carried out to compare the performance of different routing protocols in 
homogenous and heterogeneous networks. All simulated protocols misbehave in heterogeneous networks. They also suffer from high delays and achieve very low PDR. Current MANET routing protocols have unidirectional link problem and do not scale well if node density is increasing. This shows that the current routing protocols for MANET are inadaptable for heterogeneous networking. More works needs to be carried out to investigate the problems that rise with routing protocols in heterogeneous networking. In addition, most publications of routing issues in heterogeneous MANET proposed to assign most routing loads to powerful nodes. However, those nodes may create critical problems if they go off-line. Hence, there should be alternative strategies to recover any fault on powerful nodes.

\section{REFERENCES}

1. Zhenzhen, Y. and H. Yingbo. Networking by parallel relays: diversity, lifetime and routing overhead. 2004.

2. Zhenzhen, Y. and H. Yingbo. Stability of wireless relays in mobile ad hoc networks. 2005.

3. Erik, W., et al., Improving routing performance in wireless ad hoc networks using cross-layer interactions. Ad Hoc Netw., 2007. 5(5): p. 579-599.

4. Khan, A.Y., S. Rashid, and A. Iqbal. Mobility vs. predictive MPR selection for mobile ad hoc networks using OLSR. 2005.

5. Valera, A., W.K.G. Seah, and S.V. Rao. Cooperative packet caching and shortest multipath routing in mobile ad hoc networks. 2003.

6. Athanasios, B., et al., A mobility sensitive approach for efficient routing in ad hoc mobile networks, in Proceedings of the 9th ACM international symposium on Modeling analysis and simulation of wireless and mobile systems. 2006, ACM Press: Terromolinos, Spain.

7. Mueller, S. and D. Ghosal. Analysis of a distributed algorithm to determine multiple routes with path diversity in ad hoc networks. 2005.

8. Xiaojiang, D. and W. Dapeng, Adaptive cell relay routing protocol for mobile ad hoc networks. Vehicular Technology, IEEE Transactions on, 2006. 55(1): p. 278-285.

9. Ioannis, C., et al., An Experimental Study of Basic Communication Protocols in Ad-hoc Mobile Networks, in Proceedings of the 5th International Workshop on Algorithm Engineering. 2001, SpringerVerlag.

10. Ioannis, C., K. Elena, and E.N. Sotiris, On the effect of user mobility and density on the performance of protocols for ad-hoc mobile networks: Research Articles. Wirel. Commun. Mob. Comput., 2004. 4(6): p. 609-621.

11. Per, J., et al., Scenario-based performance analysis of routing protocols for mobile ad-hoc networks, in Proceedings of the 5th annual ACM/IEEE international conference on Mobile computing and networking. 1999, ACM Press: Seattle, Washington, United States.

12. Laurent, V., J. Philippe, and C. Thomas Heide, Analyzing control traffic overhead versus mobility and data traffic activity in mobile AdHoc network protocols. Wirel. Netw., 2004. 10(4): p. 447-455.

13. Abolhasan, M., J. Lipman, and J. Chicharo. A routing strategy for heterogeneous mobile ad hoc networks. in Emerging Technologies:
Frontiers of Mobile and Wireless Communication, 2004. Proceedings of the IEEE 6th Circuits and Systems Symposium on 2004.

14. Villasenor-Gonzalez, L., G. Ying, and L. Lament, HOLSR: $a$ hierarchical proactive routing mechanism for mobile ad hoc networks. Communications Magazine, IEEE, 2005. 43(7): p. 118-125.

15. Du, X. and D. Wu. Efficient multi-class routing protocol for heterogeneous mobile ad hoc networks. 2005.

16. Wei, L., et al. DELAR: device/energy/load aware relaying in heterogeneous wireless ad hoc networks. 2004.

17. Abolhasan, M., T. Wysocki, and E. Dutkiewciz, A review of routing protocols for mobile ad hoc networks. Elsevier journal of Ad hoc Networks, 2004. 12(1): p. 1-22.

18. Xiaoyan, H., X. Kaixin, and M. Gerla, Scalable routing protocols for mobile ad hoc networks. Network, IEEE, 2002. 16(4): p. 11-21.

19. Shree, M. and J.J. Garcia-Luna-Aceves, A routing protocol for packet radio networks, in Proceedings of the 1st annual international conference on Mobile computing and networking. 1995, ACM Press: Berkeley, California, United States.

20. Gerla, M., Fisheye state routing protocol (FSR) for ad hoc network. 2002, IETF Internet Draft

21. Royer, E.M. and T. Chai-Keong, A review of current routing protocols for ad hoc mobile wireless networks. Personal Communications, IEEE [see also IEEE Wireless Communications], 1999. 6(2): p. 46-55.

22. Perkins, C., E. Belding-Royer, and S. Das, Ad hoc On-Demand Distance Vector (AODV) Routing. 2003: RFC Editor.

23. Johnson, D., Y. Hu, and D. Maltz, The Dynamic Source Routing Protocol (DSR) for Mobile Ad Hoc Networks for IPv4. February 2007: RFC Editor

24. Young-Bae, K. and H.V. Nitin, Location-aided routing (LAR) in mobile ad hoc networks. Wirel. Netw., 2000. 6(4): p. 307-321.

25. Seung-Chul, M.W. and S. Suresh, Scalable routing protocol for ad hoc networks. Wirel. Netw., 2001. 7(5): p. 513-529.

26. Rajmohan, R., Topology control and routing in ad hoc networks: a survey. SIGACT News, 2002. 33(2): p. 60-73.

27. Sholander, P., et al. Experimental comparison of hybrid and proactive MANET routing protocols. 2002.

28. Samir, R.D., et al., Simulation-based performance evaluation of routing protocols for mobile ad hoc networks. Mob. Netw. Appl., 2000. 5(3): p. 179-189.

29. Ioannis, C., K. Panagiotis, and Z. Christos, Routing protocols for efficient communication in wireless ad-hoc networks, in Proceedings of the 3rd ACM international workshop on Performance evaluation of wireless ad hoc, sensor and ubiquitous networks. 2006, ACM Press: Terromolinos, Spain.

30. Ashwini, K.P. and F. Hiroshi, Study of MANET routing protocols by GloMoSim simulator. Int. J. Netw. Manag., 2005. 15(6): p. 393-410.

31. Glomosim scalable simulation environment for wireless and wired network systems. 2003 [cited; Available from: http://pcl.c.ucla.edu/sprojects/glomosim/. 